\title{
The CPT-Theorem in Two-dimensional Theories of Local Observables
}

\author{
H. J. Borchers \\ Institut für Theoretische Physik, Universität Göttingen, Bunsenstrasse 9, W-3400 Göttingen, \\ Federal Republic of Germany
}

Received January 23, 1991

\begin{abstract}
Let $\mathscr{M}$ be a von Neumann algebra with cyclic and separating vector $\Omega$, and let $U(a)$ be a continuous unitary representation of $\mathbf{R}$ with positive generator and $\Omega$ as fixed point. If these unitaries induce for positive arguments endomorphisms of $\mathscr{M}$ then the modular group act as dilatations on the group of unitaries. Using this it will be shown that every theory of local observables in two dimensions, which is covariant under translations only, can be imbedded into a theory of local observables covariant under the whole Poincare group. This theory is also covariant under the CPT-transformation.
\end{abstract}

\section{Introduction}

The CPT-theorem played an important role in relativistic quantum field theory. In the beginning of the fifties Schwinger [22] observed that there is an extra symmetry in Lagrange field theory which is now called the CPT-symmetry, i.e. a combination of space-time reflection and charge conjugation. However, this symmetry was not a symmetry in the usual sense, because Schwinger had to reverse the order of factors in the products of field operators. Three years later G. Lüders $[16,17]$ showed that the CPT-operation is, indeed, an exact symmetry of Lagrange field theory following from the Lorentz-symmetry and the standard assumptions of commutativity and the requirement that the Lagrange-function must formally be selfadjoint. For a detailed discussion see W. Pauli [19]. In 1957 R. Jost [14] gave a proof of the CPT-theorem in the frame of Wightman field theory [26] in which he revealed the connection of the CPT-symmetry with the assumptions of positivity of the energy, Lorentz-invariance, and the standard locality assumptions. In this proof the existence of a vacuum state was essential. But up to now there is no proof of the CPT-theorem in the theory of local observables in the sense of Araki, Haag and Kastler.

Initiated by the result of $\mathbf{R}$. Jost also many results about the connection of spin with statistics have been obtained in the Wightman frame, large parts of 
which are presented in the book by Streater and Wightman [23]. In the theory of local observables the question about the connection of spin with statistics went along another line because charged fields are not the fundamentals of this theory and they have to be constructed. The first result in that respect has been obtained by Borchers [3]. Some years later the subject has been re-investigated and generalized by Doplicher, Haag and Roberts $[10,11]$ and again some years later by Buchholz and Fredenhagen [9]. In these investigations no CPT-symmetry was required. However, there was one question for which the CPT-symmetry would have been useful, namely, for the existence of anti-particles. K. Fredenhagen [12] succeeded to prove their existence without using the CPT-invariance.

An absolutely different aspect of the CPT-operator has been realized in connection with the Tomita [25] Takesaki [24] theory of modular Hilbert-algebras. Bisognano and Wichmann [1,2] observed that the CPT-operator and the Lorentzboosts are related to the modular conjugation and to the modular group of the wedge algebra (see Sect. IV for its definition), provided the theory of local observables is generated from a Wightmann field which is covariant under Lorentztransformations. Here, again, one is dealing with the vacuum-sector.

The result of Bisognano and Wichmann shows that the modular group of the wedge algebra coincided with the Lorentz-boosts of the wedge. This result held out hopes for some converse conclusion to be true, namely, that the modular group of the algebra, associated to the wedge domain, can indeed be identified with a Lorentz-boost. This hope has been supported by a recent result of the author [5] showing that there is a close connection between the translations and the modular operator for local regions.

In this paper we want to show that the modular group for the algebra of the wedge domain can always be interpreted as the group of Lorentz-boosts, provided the cyclic and separating vector is the vacuum-vector. In order to obtain this result one must show that the modular group induces outer automorphisms of the translation group. To prove this two properties are essential. One is the spectrum condition for the translations, the other is the structure of the wedge domain, which is mapped by a semi-group of translations into itself. If the wedge is fixed then one is dealing with a two-dimensional problem. Therefore, in most of the investigations we shall only deal with field theories in two space-time dimensions. Also in this situation the construction of the Lorentz-group is not unique because one can start either with the right wedge or the left wedge. Only if the algebra of the left wedge is the commutant of the algebra of the right wedge then the constructed Lorentz-group is unique. If we don't have this identity then one can construct two local nets containing the original net and both fulfilling the duality condition. The construction will be done by a method due to Bisognano and Wichmann. We call these nets Bisognano-Wichmann-nets. If the original net happens to be a Lorentz-covariant net then the given Lorentz-transformations and those constructed from the modular group need not to coincide. If they are different then the difference gives rise to a local gauge. Such situations might appear when considering Wightman fields with an infinite number of components.

All of our investigations are essentially two-dimensional. This implies that we can prove a CPT-theorem only for the theory of local observables in two dimensions. An extension to higher dimensions needs additional information about the action of the modular group on sub-algebras. Such information is not known to me. 
Using the information about the two dimensional situation one is able to construct for every element of the Lorentz group unitary operators inducing on the translations the correct automorphisms. But we do not know whether or not we can choose a family which also forms a representation of the Lorentz group. The main question however remains the problem of the locality of the action in higher dimensions.

\section{Some General Results}

In this section we will derive some general results which are interesting by themselves and which will be used in the following sections. We assume that $\mathscr{M}$ is a von Neumann-algebra acting on a Hilbert-space $\mathscr{H}$ and that $\Omega \in \mathscr{H}$ is cyclic and separating for $\mathscr{M}$. The modular operator and the modular conjugation associated with the pair $\{\mathscr{M}, \Omega\}$ will be denoted by $\Delta$ and $J$, respectively. First we show

II.1. Lemma. Let $\{\mathscr{M}, \mathscr{H}, \Omega, \Delta, J\}$ be as described above. Assume, moreover, $U(t) \in \mathscr{B}(\mathscr{H})$ is a continuous unitary one parametric group with

(i) $\operatorname{ad} U(t) \mathscr{M}=U(t) \mathscr{M} U^{*}(t)=\mathscr{M} \forall t$.

(ii) $U(t) \Omega=\Omega \forall t$.

(iii) $U(t)$ has an analytic extension into the upper t-halfplane.

Then $U(t)$ is entire analytic.

If, moreover, $U(t)$ has a positive generator then $U(t) \equiv 1$.

Proof. From the assumptions one knows that $U(t)$ commutes with $\Delta$ as well as with $J$, [6] Theorem 3.2.18. We will make use of the latter fact. For $\psi, \varphi \in \mathscr{H}$ we have

$$
(\psi, U(t) \varphi)=(\psi, J U(t) J \varphi)=(J \varphi, U(-t) J \psi) .
$$

Now the left-hand side has an analytic extension into the upper halfplane and the right-hand side has an extension into the lower halfplane. Since both sides coincide on the real points, $(\psi, U(t) \varphi)$ extends to an entire analytic function. Since this holds for arbitrary $\varphi, \psi \in \mathscr{H}$, it follows that $U(t)$ itself is entire analytic.

If $U(t)$ has a positive generator, which must have the eigenvalue zero, then $(\psi, U(t) \varphi)$ is bounded on the upper halfplane and $(J \varphi, U(-t) J \psi)$ is bounded on the lower halfplane. Since a bounded entire analytic function is constant one obtains $(\psi, U(t) \varphi)=(\psi, \varphi)$, which implies $U(t)=1$.

From this result we learn that a von Neumann-algebra $\mathscr{M}$, with cyclic and separating vector $\Omega$, cannot have an invariance group for which $\omega_{\Omega}$ is an invariant state and for which the canonical group-representation fulfills the spectrum condition. This is not true if we only require ad $U(t) \mathscr{M} \subset \mathscr{M}$ for $t>0$. An example is the "wedge algebra" in the theory of local observables. Here one has to take the translation along the lightcone coordinate appearing in the definition of the wedge and as Hilbert-space that of the vacuum sector. Before starting these investigations we show

II.2. Lemma. Let $\{\mathscr{M}, \mathscr{H}, \Omega, \Delta, J\}$ be as described before. Assume $U \in B(\mathscr{H})$ is unitary, such that $U \mathscr{M} U^{*} \subset \mathscr{M}$ and $U \Omega=\Omega$, then one has with $S=J \Delta^{1 / 2}$ the relation

$$
U S=S U \text {. }
$$


Proof. For $A \in \mathscr{M}$ it follows that both vectors $A \Omega$ and $U A \Omega=U A U^{*} \Omega$ belong to the domain of $S$ and one obtains

$$
S U A \Omega=S U A U^{*} \Omega=U A^{*} U^{*} \Omega=U A^{*} \Omega=U S A \Omega .
$$

Assume now $\left\{A_{n} \Omega\right\}$ converges in the graph topology of $S$. This means the sequence $\left\{A_{n} \Omega, A_{n}^{*} \Omega\right\}$ converges in $\mathscr{H} \oplus \mathscr{H}$ to a vector $\left\{\psi, \psi^{*}\right\}$, and one has $S \psi=\psi^{*}$. Multiplying this with $U$ we see that also $\left\{U A_{n} \Omega, U A_{n}^{*} \Omega\right\}$ converges to $\left\{U \psi, U \psi^{*}\right\}$. This shows $U D_{S} \subset D_{S}$ and it follows

$$
S U \psi=\lim _{n} S U A_{n} \Omega=\lim _{n} U A_{n}^{*} \Omega=U \psi^{*}=U S \psi
$$

As a consequence of this lemma we show next:

II.3. Lemma. Let $\{\mathscr{M}, \mathscr{H}, \Omega, U, \Delta, J\}$ be as in Lemma II.2. Then the operator

$$
\Delta^{t t} U \Delta^{-t}
$$

has an analytic extension into the strip,

$$
S\left(-\frac{1}{2}, 0\right)=\left\{z ;-\frac{1}{2}<\mathfrak{I} m z<0\right\}
$$

which is continuous on the boundary fulfilling the estimate

$$
\left\|\Delta^{\imath z} U \Delta^{-\imath z}\right\| \leqq 1 \text {. }
$$

Proof. If $\psi, \varphi \in D(\Delta) \cap D\left(\Delta^{-1}\right)$ then $\left(\psi, \Delta^{i z} U \Delta^{-l z} \varphi\right\}$ is defined on the closed strip and analytic in the interior. Moreover, it is bounded by $\left\|\Delta^{1 / 2} \psi\right\|\left\|\Delta^{-1 / 2} \varphi\right\|$. Putting now $z=t-\frac{l}{2}$, we obtain

$$
\begin{aligned}
\left(\psi, \Delta^{t t} \Delta^{1 / 2} U \Delta^{-1 / 2} \Delta^{-t t} \varphi\right) & =\left(\psi, \Delta^{t t} J S U \Delta^{-1 / 2-t t} \varphi\right) \\
& =\left(\psi, \Delta^{u t} J U J \Delta^{-t t} \varphi\right)
\end{aligned}
$$

Since, now, a bounded function, analytic in a strip, takes its maximum value of its modulus on the boundary we obtain

$$
\left(\psi, \Delta^{t z} U \Delta^{-t z} \varphi\right) \leqq\|\psi\|\|\varphi\| .
$$

Since $\psi$ and $\varphi$ are running through a dense set of vectors it follows that $\Delta^{i z} U \Delta^{-l z}$ has an extension as a bounded operator valued function with norm not exceeding 1.

II.4. Remarks.

(i) Since $U$ is unitary one has $U^{*} \mathscr{M}^{\prime} U \subset \mathscr{M}^{\prime}$ because of the equation $U^{*} A^{\prime} U A=$ $U^{*} A^{\prime} U A U^{*} U=U^{*} U A U^{*} A^{\prime} U=A U^{*} A^{\prime} U$.

(ii) If one has in addition $U^{*} \Omega=\Omega$ we obtain from Lemma II.1 the equation

$$
F U^{*}=U^{*} F \text {. }
$$

According to Lemma II.2 the expression

$$
\Delta^{i t} U^{*} \Delta^{-i t}
$$

has an analytic continuation into the strip $S\left(0, \frac{1}{2}\right)$ and fulfills the estimate

$$
\left\|\Delta^{z z} U^{*} \Delta^{-l z}\right\| \leqq 1 \text { for } z \in S\left(0, \frac{1}{2}\right) \text {. }
$$


Next we assume that we have a continuous unitary group $U(a)$ which fulfills spectrum condition and which leaves the cyclic and separating vector $\Omega$ invariant. Moreover, we shall assume that the semi-group ad $U(a), a \geqq 0$ maps the algebra $\mathscr{M}$ into itself. Remark that $\mathscr{M}$ is not invariant under the whole group if we assume that $U(a)$ is not trivial (see Lemma II.1).

For the following investigation we shall replace the variable $a$ by $e^{\xi}$, which has the advantage that $a>0$ is replaced by $\xi \in \mathbf{R}$ and the upper half-plane $\mathfrak{I} m a>0$ by the strip $0<\mathfrak{I} m \xi<\pi$. The boundary of this strip at $\mathfrak{I} m \varphi=\pi$ corresponds to the values $a<0$. Now we show

II.5. Proposition. Let $\{\mathscr{M}, \mathscr{H}, \Omega, \Delta, J\}$ fulfill the assumptions described above. Assume $U(a), a \in \mathbf{R}$ is a continuous unitary group, $U(a) \in \mathscr{B}(\mathscr{H})$, fulfilling the properties

(i) $\operatorname{ad} U(a) \mathscr{M} \subset \mathscr{M}$ for $a \geqq 0$.

(ii) $U(a) \Omega=\Omega$.

(iii) The generator of $U(a)$ is positive, then

1. The expression

$$
\Delta^{t t} U\left(e^{\xi}\right) \Delta^{-t t}=: V(t, \xi)
$$

has an analytic extension into the tube $T$ and is continuous on the closure, where $T$ is defined as follows:

$$
T=\{(z, \zeta) ;(\mathfrak{I} m z, \mathfrak{I} m \zeta) \in B\},
$$

where $B$ is the interior of the quadrangle, whose corners are given by the point

$$
\left\{(0,0),\left(-\frac{1}{2},-\pi\right),\left(-\frac{1}{2}, 0\right),(0, \pi)\right\} \text {. }
$$

2. The operator $V(z, \zeta)$ is bounded

$$
\|V(z, \zeta)\| \leqq 1, \text { for } \quad(z, \zeta) \in T .
$$

3. $V\left(t-\frac{l}{2}, \xi-\imath \pi\right)$ has the value

$$
V\left(t-\frac{l}{2}, \xi-i \pi\right)=\Delta^{t t} J U\left(-e^{\xi}\right) J \Delta^{-\imath t}
$$

Proof. If $\psi, \varphi \in D(\Delta) \cap D\left(\Delta^{-1}\right)$ then we consider the function

$$
\left(\psi, \Delta^{i z} U\left(e^{\zeta}\right) \Delta^{-l z} \varphi\right)=: F(z, \zeta)
$$

which is analytic and bounded in

$$
(z, \zeta) \in S\left(-\frac{1}{2}, 0\right) \times S(0, \pi)
$$

But, by the spectrum condition, for $z$ real $\left\|U\left(e^{\zeta}\right)\right\|$ is bounded by 1 . For $\zeta=\xi$ real we know from Lemma II.3 that $\left\|\Delta^{i z} U(\xi) \Delta^{-i z}\right\| \leqq 1$ for $z \in S\left(-\frac{1}{2}, 0\right)$. This implies $|F(z, \zeta)| \leqq\|\psi\|\|\varphi\|$ if either $z$ or $\zeta$ is real. Hence we obtain by analytic completion the estimate

$$
F(z, \zeta) \leqq\|\psi\|\|\varphi\|
$$

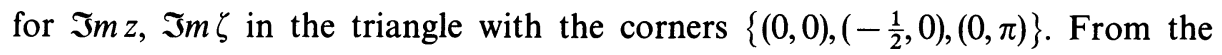


proof of Lemma II.3 we know

$$
F\left(t-\frac{l}{2}, \xi\right)=\left(\psi, \Delta^{i t} J U\left(e^{\xi}\right) J \Delta^{-i t} \varphi\right)
$$

Since $J$ is an anti-unitary operator and since $U\left(e^{\xi}\right)$ has an analytic extension into the strip $S(0, \pi)$ we see that $J U\left(e^{\zeta}\right) J$ is anti-analytic in that strip, which means that $J U\left(e^{\bar{\zeta}}\right) J$ is analytic in $S(-\pi, 0)$. This can also be seen by direct calculation:

$$
F\left(t-\frac{l}{2}, \xi\right)=\overline{\left(J \psi, \Delta^{t t} U\left(e^{\xi}\right) \Delta^{-t t} J \varphi\right)}=\left(J \varphi, \Delta^{t t} U\left(-e^{\xi}\right) \Delta^{-t t} J \psi\right)
$$

which has in $\xi$ an analytic continuation into $S(-\pi, 0)$ with the estimate

$$
\left|F\left(t-\frac{l}{2}, \zeta\right)\right| \leqq\|\psi\|\|\varphi\| \text {. }
$$

Since we have chosen $\varphi$ and $\psi$ in $D(\Delta) \cap D\left(\Delta^{-1}\right)$ the function has an analytic extension into $S\left(0, \frac{1}{2}\right) \times S(-\pi, 0)$ or $F(z, \zeta)$ into $S\left(-\frac{1}{2}, 0\right) \times S(-\pi, 0)$. But this function is bounded by $\|\psi\|\|\varphi\|$ on the boundaries of the degenerated tubes $S\left(-\frac{1}{2}, 0\right) \times\{0\} \cup\left\{-\frac{1}{2}\right\} \times S(-\pi, 0)$, and hence on the tube which has as basis the triangle defined by the points $\left\{(0,0),\left(-\frac{1}{2},-\pi\right),\left(-\frac{1}{2}, 0\right)\right\}$. Together with the result of Lemma II.3 we find that $F(z, \zeta)$ is analytic in the tube $T$ defined in Eq. (6) and (6a) and bounded in this domain by

$$
|F(z, \zeta)| \leqq\|\psi\|\|\varphi\|
$$

Hence the operator $V(z, \zeta)$ is an analytic operator in the tube $T$ and fulfills the estimate

$$
\|V(z, \zeta)\| \leqq 1
$$

Finally one has $F\left(t-\frac{l}{2}, \xi-\imath \pi\right)=\left(\psi, \Delta^{\imath t} J U\left(-e^{\xi}\right) J e^{-\imath t} \varphi\right)$ which implies

$$
V\left(t-\frac{l}{2}, \xi-\imath \pi\right)=\Delta^{t t} J U\left(-e^{\xi}\right) J \Delta^{-t t}
$$

This proves the proposition.

\section{II.6. Addendum.}

(a) Assume for $\{\mathscr{M}, \mathscr{H}, \Omega, \Delta, J\}$ the same situation as before and let $U(a)$ be again a unitary group fulfilling spectrum condition which leaves the vector $\boldsymbol{\Omega}$ invariant. Instead of looking at the situation investigated above we consider

$$
\operatorname{ad} U(a) \mathscr{M}^{\prime} \subset \mathscr{M}^{\prime} \text { for } a \leqq 0 .
$$

In this case we know from the Remark II. 4 that $F U(a)=U(a) F$ for $a \leqq 0$ holds and, moreover, that the operator

$$
W(t, \xi)=\Delta^{t t} U\left(-e^{\xi}\right) \Delta^{-t t}
$$

has in $t$ an analytic extension into the strip $S\left(0, \frac{1}{2}\right)$. 
The considerations of Lemma II.3 and of Proposition II.5 also have their transscription and one obtains

1. $W(t, \xi)$ has an analytic extension into the tube $-T$.

2. For $(z, \zeta) \in-T$ one has that $W(z, \zeta)$ is bounded

$$
\|W(z, \zeta)\| \leqq 1 .
$$

3. $W\left(t+\frac{l}{2}, \xi+\imath \pi\right)$ has the form

$$
W\left(t+\frac{l}{2}, \xi+\imath \pi\right)=\Delta^{t t} J U\left(e^{\xi}\right) J \Delta^{-\imath t} .
$$

(b) However, if we assume

$$
\operatorname{ad} U(a) \mathscr{M} \subset \mathscr{M} \text { for } a \leqq 0
$$

then $S U(a)=U(a) S$ for $a \leqq 0$ holds. If we introduce the operator

$$
\hat{V}(t, \xi)=\Delta^{t} U\left(-e^{\xi}\right) \Delta^{-\imath t}
$$

then the analytic extension of $t$ does not change, but in $\xi$ we have an analytic extension into the tube $S(-\pi, 0)$. All other considerations are similar as in the case of the operator $V(t, \xi)$. Hence we obtain

1. $\hat{V}(t, \xi)$ has an analytic extension into the tube $\hat{T}$, where $\hat{T}$ is obtained from $T$ by replacing $\zeta$ by $-\zeta$.

2. For $(z, \zeta) \in \hat{T}$ the operator $\hat{V}(z, \zeta)$ is bounded

$$
\|\hat{V}(z, \zeta)\| \leqq 1 .
$$

3. $\hat{V}\left(t+\frac{l}{2}, \xi-\imath \pi\right)$ has the form

$$
\hat{V}\left(t+\frac{l}{2}, \xi-\imath \pi\right)=\Delta^{t t} J U\left(+e^{\xi}\right) J \Delta^{-t t} .
$$

Next we want to apply the results obtained in Proposition II.5 and Addendum II.6 in order to compute special matrix elements. But first we introduce some notations:

II.7. Definition. Let $\{\mathscr{M}, \mathscr{H}, \Omega, \Delta, J\}$ be as before and let $A \in \mathscr{M}$ and $B \in \mathscr{M}^{\prime}$ then we define:

$$
F^{+}(t, \xi)=\left(\Omega, B \Delta^{t t} U\left(e^{\xi}\right) \Delta^{-t t} A \Omega\right)
$$

and

$$
F^{-}(t, \xi)=\left(\Omega, A \Delta^{t t} U\left(-e^{\xi}\right) \Delta^{-t t} B \Omega\right) .
$$

From the result of Proposition II.5 we know that $F^{+}(t, \xi)$ has a bounded analytic extension into the tube $T$ and by Addendum II.6 we know that $F^{-}$has an extension into the tube $-T$.

Next we show

II.8. Proposition. The two functions $F^{+}(t, \xi)$ and $F^{-}(t, \xi)$, which are holomorphic in the tubes $T$ and $-T$ respectively, are different representations of one analytic function 
$H(z, \xi)$, which is holomorphic in the domain

$$
\left\{\mathfrak{R} z, \mathfrak{R} e \zeta \in \mathbf{R}^{2}\right\} \cap\{-\pi<2 \pi \mathfrak{I} m z-\mathfrak{I} m \zeta<\pi\} .
$$

Here $H(z, \zeta)$ is bounded. One has

$$
|H(z, \zeta)| \leqq \max \left\{\|A \Omega\|\left\|B^{*} \Omega\right\|,\left\|A^{*} \Omega\right\|\|B \Omega\|\right\} .
$$

This latter statement implies that $H(z, \zeta)$ is constant along the direction given by the strip (8) i.e.

$$
H(z, \zeta)=H\left(z+\frac{w}{2}, \zeta+w \tau\right) \text { for } \quad w \in \mathbf{C} .
$$

Proof. Since the modular automorphism maps $\mathscr{M}$ into itself one has that

$$
\operatorname{ad} \Delta^{t t} U\left(e^{\xi}\right) e^{-t t} \mathscr{M} \subset \mathscr{M}
$$

for $(t, \xi) \in \mathbf{R}^{2}$, which implies that ad $\Delta^{t t} U\left(e^{\xi}\right) \Delta^{-t t} A$ commutes with $B$. This implies that $F^{+}(t, \xi)=F^{-}(t, \xi)$ for $(t, \xi) \in \mathbf{R}^{2}$. Using the edge of the wedge theorem [7], [8] we see that both functions coincide and have, by the tube-theorem, an analytic extension into the convex hull of $T \cup-T . F^{+}$is bounded by $\left\|B^{*} \Omega\right\|\|A \Omega\|$ and $F^{-}$is bounded by $\left\|A^{*} \Omega\right\|\|B \Omega\|$. This implies $H$ is bounded by the maximum of the two estimates. From Proposition II.5 we know

$$
F^{+}\left(t-\frac{l}{2}, \xi-\imath \pi\right)=\left(\Omega, B \Delta^{t t} J U\left(-e^{\xi}\right) J A \Omega\right)
$$

and from Addendum II.6 we obtain

$$
F^{-}\left(t+\frac{l}{2}, \xi+\imath \pi\right)=\left(\Omega, A \Delta^{t t} J U\left(e^{\xi}\right) J B \Omega\right) .
$$

Since $J \mathscr{M} J=\mathscr{M}^{\prime}$ it follows that

$$
\operatorname{ad} J U\left(-e^{\xi}\right) J \mathscr{M} \subset \mathscr{M}
$$

which implies

$$
F^{+}\left(t-\frac{l}{2}, \xi-\imath \pi\right)=F^{-}\left(t+\frac{l}{2}, \xi+\imath \pi\right) .
$$

This implies that the common function $H(z, \zeta)$ is a periodic function

$$
H(z, \zeta)=H(z+\imath, \zeta+\imath \pi) \text {. }
$$

Using again the edge of the wedge theorem we see that $H(z, \zeta)$ is analytic in the tube described in (8). Since $H(z, \zeta)$ is also bounded in this domain by $\max \left\{\left\|B^{*} \Omega\right\|\|A \Omega\|\right.$, $\left.\left\|A^{*} \Omega\right\|\|B \Omega\|\right\}$ it must be constant in the direction of periodicity. This proves the theorem.

Next we want to apply the result of Proposition II.8 in order to obtain the main result of this section. For this we make use of the assumption that $\Omega$ is cyclic and separating for $\mathscr{M}$.

II.9. Theorem. Let $\mathscr{M}$ be a von Neumann-algebra acting on a Hilbert-space $\mathscr{H}$ and assume $\Omega \in \mathscr{M}$ is cyclic and separating for $\mathscr{M}$. Denote the modular operator and 
modular conjugation of the pair $\{\mathscr{M}, \Omega\}$ by $\Delta, J$. Let $U(a), a \in \mathbf{R}$ be a continuous unitary one parametric group with a positive generator leaving the vector $\Omega$ invariant.

(A) Assume in addition

$$
\operatorname{ad} U(a) \mathscr{M} \subset \mathscr{M} \quad \text { for } \quad a \geqq 0
$$

then we have for $(t, a) \in \mathbf{R}^{2}$

(a)

and

$$
\Delta^{t t} U(a) \Delta^{-t t}=U\left(e^{-2 \pi t} a\right)
$$

(b)

$$
J U(a) J=U(-a) .
$$

(B) If we assume instead

$$
\operatorname{ad} U(a) \mathscr{M} \subset \mathscr{M} \quad \text { for } \quad a \leqq 0
$$

then we have for $(t, a) \in \mathbf{R}^{2}$ :

(a)

and

$$
\Delta^{t t} U(a) \Delta^{-t t}=U\left(e^{2 \pi t} a\right)
$$

(b)

$$
J U(a) J=U(-a) .
$$

Proof.

(A) For $A \in \mathscr{M}$ and $B \in \mathscr{M}^{\prime}$ and with $V(t, \xi)=\Delta^{t t} U\left(e^{\xi}\right) \Delta^{-t t}$ we have by Proposition II.8 the equation

$$
(\Omega, B V(t, \xi) A \Omega)=(\Omega, B V(t+s, \xi+2 \pi s) A)
$$

for arbitrary $s \in \mathbf{C}$. Since $\Omega$ is cyclic and separating and since the operators in question are bounded we conclude

$$
V(t, \xi)=V(t+s, \xi+2 \pi s)
$$

Choosing $s=-t$ we obtain

$$
\Delta^{t t} U\left(e^{\xi}\right) \Delta^{-\imath t}=U\left(e^{-2 \pi t} e^{\xi}\right)
$$

and inserting $e^{\xi}=a$ we have

$$
\Delta^{t t} U(a) \Delta^{-t t}=U\left(e^{-2 \pi t} a\right) \text { for } a \geqq 0 .
$$

Since both sides of this equation are boundary values of analytic operator valued functions in the upper half-plane we obtain Eq. (10) by analytic continuation.

For the statement (b) we make use of Eq. (4) which reads

$$
J U\left(e^{\xi}\right) J=\Delta^{1 / 2} U\left(e^{\xi}\right) \Delta^{-1 / 2}=V\left(-\frac{l}{2}, \xi\right) .
$$

But by the invariance property of $V$ we obtain

$$
V\left(-\frac{l}{2}, \xi\right)=V(0, \xi+\imath \pi)=U\left(e^{\imath \pi} e^{\xi}\right)=U\left(-e^{\xi}\right)
$$


or $J U(a) J=U(-a)$ for $a \geqq 0$. Taking the adjoint of this equation we obtain Eq.(11). (B) For proving these statements we start from part (b) of Addendum II.6 which indicates that there is a similar result as Proposition II.8 with the difference that we obtain invariance of the form

$$
\hat{H}(z, \zeta)=\hat{H}\left(z+\frac{w}{2}, \zeta-\pi w\right) \text { for } w \in \mathbf{C} .
$$

From this we obtain for the operator $\hat{V}(t, \xi)=\Delta^{t t} U\left(-e^{\xi}\right) \Delta^{-t t}$ the relation

$$
\hat{V}(t, \xi)=\hat{V}(t+s, \xi-2 \pi s)
$$

Repeating the same steps as in the proof of (A) we obtain the statements of (B).

\section{Two-Dimensional Theories}

In this section we assume again that we have a von Neumann-algebra $\mathscr{M}$ acting on a Hilbert-space $\mathscr{H}$ such that there is a vector $\Omega \in \mathscr{H}$, which is cyclic and separating for $\mathscr{M} . \Delta$ and $J$ will denote again the modular conjugation of the pair $\{\mathscr{M}, \Omega\}$. But now we assume that we have a continuous representation $U(a), a \in \mathbf{R}^{2}$ of the vector group of $\mathbf{R}^{2}$. Furthermore, we assume

(a) $U(a) \Omega=\Omega$.

(b) The Spectrum of $U(a)$ is contained in the forward light cone $V^{+}:=\left\{a \in \mathbf{R}^{2} ; a_{0} \geqq\right.$ $\left.\left|a_{1}\right|\right\}$.

(c) Let $W$ be the set

$$
W:=\left\{a \in \mathbf{R}^{2} ; a_{1} \geqq\left|a_{0}\right|\right\}
$$

then we assume

$$
\operatorname{ad} U(a) \mathscr{M} \subset \mathscr{M} \text { for } \quad a \in W .
$$

Now we introduce the lightcone coordinates

$$
a^{+}=\frac{a_{0}+a_{1}}{2}, a^{-}=\frac{a_{0}-a_{1}}{2} \text { or } a_{0}=a^{+}+a^{-}, a_{1}=a^{+}-a^{-} .
$$

Since $U(a)$ fulfills the spectrum condition it follows that $U\left(a^{+}\right)$and $U\left(a^{-}\right)$both have positive generators and, moreover, we have

$$
\begin{aligned}
& \text { ad } U\left(a^{+}\right) \mathscr{M} \subset \mathscr{M} \text { for } a^{+} \geqq 0 \text { and } \\
& \operatorname{ad} U\left(a^{-}\right) \mathscr{M} \subset \mathscr{M} \text { for } a^{-} \leqq 0 \text {. }
\end{aligned}
$$

Therefore, we apply Theorem II.9 and obtain

III.1. Theorem. With the assumptions described in the beginning of this section and with

$$
\Lambda(t)=\left(\begin{array}{cc}
\cosh 2 \pi t & -\sinh 2 \pi t \\
-\sinh 2 \pi \mathrm{t} & \cosh 2 \pi t
\end{array}\right)
$$


and

$$
U(\Lambda(t))=\Delta^{t t},
$$

then $\{U(\Lambda), U(a)\}$ induce a representation of the two-dimensional Poincaré-group which fulfills the spectrum condition and which has $\Omega$ as invariant vector. Furthermore, one has

$$
J U(a) J=U(-a) .
$$

Proof. From Theorem II.9 we know $\Delta^{t t} U\left(a^{+}\right) \Delta^{-t t}=U\left(e^{-2 \pi t} a^{+}\right)$and $\Delta^{t t} U\left(a^{-}\right) \Delta^{-t t}=$ $U\left(e^{2 \pi t} a^{-}\right)$. This together gives $\Delta^{t t} U(a) \Delta^{-t t}=U(\Lambda(t) a)$, where $\Lambda(t)$ is given by Eq. (12). This means $U(\Lambda)$ induces the correct outer automorphism on the translation group. This implies that the above system gives a representation of the Poincare-group. The statement of Eq. (14) has been proved in Theorem II.9.

Remark. In the investigations of Bisognano and Wichmann [1, 2] appears also a relation between the Lorentz-boosts and the modular group $\sigma_{t}$, which differs by a sign. This is due to the fact that the modular automorphism is often defined by the equation $\sigma_{t}(A)=\operatorname{ad} \Delta^{-t} A$, while we are working with ad $\Delta^{t t}$.

If one is dealing with a theory of local observables then it has been shown by Borchers [3] that the spectrum is located in a Lorentz invariant set. At first sight it seems as if the above results do not use any locality conditon. That this is not true will be shown by the next investigation.

III.2. Lemma. With the assumptions described in the beginning of this section and with

$$
\mathscr{M}_{a}=U(a) \mathscr{M} U(-a), \quad \mathscr{M}_{a}^{\prime}=U(a) \mathscr{M}^{\prime} U(-a)
$$

one has with the notations (12) and (13)

(i) $\left(\mathscr{M}_{a}\right)^{\prime}=\mathscr{M}_{a}^{\prime}$,

(ii) $U(\Lambda) \mathscr{M}_{a} U^{*}(\Lambda)=\mathscr{M}_{\Lambda a}$,

$$
U(\Lambda) \mathscr{M}_{a}^{\prime} U^{*}(\Lambda)=\mathscr{M}_{\Lambda a}^{\prime}
$$

(iii) $J \mathscr{M}_{a} J=\mathscr{M}_{-a}^{\prime}$ and ${ }^{\Lambda a} \mathscr{M}_{a}^{\prime} J=J \mathscr{M}_{-a} J$.

Proof. The first statement is trivial. Since $U(\Lambda)$ is up to some scaling of the parameters identical with the modular group, we have ad $U(\Lambda) \mathscr{M}=\mathscr{M}$ and ad $U(\Lambda) \mathscr{M}^{\prime}=\mathscr{M}^{\prime}$. Hence we obtain by definition (15) and Theorem III.1,

$$
\begin{aligned}
U(\Lambda) \mathscr{M}_{a} U^{*}(\Lambda) & =U(\Lambda) U(a) \mathscr{M} U^{*}(a) U^{*}(\Lambda)=U(\Lambda a) U(\Lambda) \mathscr{M} U^{*}(\Lambda) U^{*}(\Lambda a) \\
& =U(\Lambda a) \mathscr{M} U^{*}(\Lambda a)=\mathscr{M}_{\Lambda a} .
\end{aligned}
$$

The arguments for $\mathscr{M}_{a}^{\prime}$ and for the statement (iii) are analogous.

Double cones are usually characterized by two points $a, b$ such that $b-a \in V^{+}$. But since we are working with two-dimensional theories one can characterize them by two points which are spacelike to each other. To this end one uses the right wedge $W_{R}$ and the left wedge $W_{L}$. We say $b$ is right of $a$ if $b \in a+W_{R}$. Let $b$ be right of $a$, then we put

$$
D_{a, b}=\left\{a+W_{R}\right\} \cap\left\{b+W_{L}\right\} .
$$

With these notations we obtain 
III.3. Proposition. With the assumptions and notations of the last lemma and with the definition (16) introduce

$$
\mathscr{N}\left(D_{a, b}\right)=\mathscr{M}_{a} \cap \mathscr{M}_{b}^{\prime}
$$

and let $\mathscr{N}$ be the $C^{*}$-algebra generated by all the $\mathscr{N}\left(D_{a, b}\right)$. Then the system $\left\{\mathscr{N}(D), \mathcal{N}, U(\Lambda, a) \mathbf{R}^{2}\right\}$, where $U(\Lambda, a)$ is the representation of the Poincaré-group acting on $\mathbf{R}^{2}$, defines a covariant local net, having $J$ as CPT-operator.

Proof. From Lemma III.2 we know the co-variance of the systems $\left\{\mathscr{M}_{a}\right\}$ and $\left\{\mathscr{M}_{a}^{\prime}\right\}$. Hence we have co-variance of the intersections. Since $J$ interchanges $\left\{\mathscr{M}_{a}\right\}$ with $\left\{\mathscr{M}_{a}^{\prime}\right\}$ in particular $J \mathscr{M}_{a} J=\mathscr{M}_{-a}^{\prime}$. Therefore, $J$ maps $\mathscr{M}\left(D_{a, b}\right)$ onto $\mathscr{N}\left(D_{-b .-a}\right)$. It remains to show the locality. If $D_{a, b}$ and $D_{c, d}$ are spacelike to each other then either $D_{c, d}$ is right of $D_{a, b}$ or vice versa. Let us assume $D_{c, d}$ is right of $D_{a, b}$ then $\mathscr{N}\left(D_{a, b}\right) \subset \mathscr{M}_{b}^{\prime}$ and $\mathscr{N}\left(D_{c, d}\right) \subset \mathscr{M}_{c}$. Since $c$ is right of $b$ we have $\mathscr{M}_{c} \subset \mathscr{M}_{b}$ and hence the algebras commute. The other case can be proved in the same manner.

Having the local net formed by the von Neumann-algebras $\mathscr{N}\left(D_{a, b}\right)$ we can construct $\mathscr{N}\left(W_{R}\right)$ and $\mathscr{N}\left(W_{L}\right)$ as the von Neumann-algebra generated by all the $\mathcal{N}\left(D_{a, b}\right)$ with $D_{a, b} \subset W_{R}$ or $D_{a, b} \subset W_{L}$, respectively. One would like to compare these algebras with $\mathscr{M}$ or $\mathscr{M}^{\prime}$, respectively. By construction we know that $\mathscr{N}\left(W_{R}\right)$ is a sub-algebra of $\mathscr{M}$ and $\mathscr{N}\left(W_{L}\right)$ is a sub-algebra of $\mathscr{M}^{\prime}$.

A comparison, however, only makes sense if the algebras are big enough. As criterium we will use the requirement that $\mathcal{N} \Omega$ is dense in $\mathscr{H}$. First we show

III.4. Lemma. Let $\left\{\mathscr{N}\left(D_{a, b}\right), \mathcal{N}, U(a), \mathbf{R}^{2}\right\}$ be a translation covariant local net and $U(a)$ a representation of the translation group fulfilling the spectrum condition acting on a Hilbert-space with vacuum vector. Then $\mathcal{N}\left(W_{R}\right) \Omega$ and $\mathcal{N}\left(W_{L}\right) \Omega$ are both dense in $\mathcal{N} \Omega$.

Proof. Let $b$ be in the interior of $W_{R}$. Then $\mathscr{N}\left(W_{R}+b\right) \subset \mathcal{N}\left(W_{R}\right)$ and there exists a neighbourhood $U$ such that $\mathcal{N}\left(W_{R}\right)=\bigcup_{a \in U} U(a) \mathcal{N}\left(W_{R}+b\right) U^{*}(a)$. But this implies by a theorem of Borchers [4] that $\overline{\mathcal{N} \Omega}=\overline{\mathcal{N}\left(W_{R}\right) \Omega}$ because $\mathcal{N}$ is the algebra generated by all $U(a) \mathcal{N}\left(W_{R}+b\right) U^{*}(a)$. The same argument also holds for $W_{L}$. After these preparations we obtain:

III.5. Theorem. Let $\mathscr{M}$ be a von Neumann-algebra such that $\{\mathscr{M}, U(a), \Omega, \mathscr{H}, \Delta, J\}$ fulfills the assumptions and conditions described in the beginning of this section. Let $\left\{\mathcal{N}\left(D_{a, b}\right), \mathcal{N}, U(\Lambda, a) J, \mathbf{R}^{2}\right\}$ be the covariant local net derived from $\{\mathscr{M}, U(a)\}$ described in Proposition III.3. If we assume that $\mathcal{N} \Omega$ is dense in $\mathscr{H}$ then we have $\mathscr{N}\left(W_{R}\right)=\mathscr{M}$ and $\mathscr{N}\left(W_{L}\right)=\mathscr{M}^{\prime}$. In particular does this imply that the local net $\left\{\mathscr{N}\left(D_{a, b}\right), \mathcal{N}, U(\Lambda, a), J, \mathbf{R}^{2}\right\}$ fulfills the duality condition.

Proof. Since $\mathscr{N} \Omega$ is dense in $\mathscr{H}$ we have, by Lemma III.4, that also $\mathcal{N}\left(W_{R}\right) \Omega$ is dense in $\mathscr{H} . \mathscr{N}\left(W_{R}\right)$ is by construction contained in $\mathscr{M} . \mathscr{N}\left(W_{R}\right)$ is invariant under ad $U(\Lambda)$. By the form $U(\Lambda(t))=\Delta^{t}$, where $\Delta$ is the modular operator of $\mathscr{M}$, we know that $\mathcal{N}\left(W_{R}\right)$ is invariant under the modular automorphisms of $\mathscr{M}$. This implies by a theorem of Rigotti [21], which is based on a result of Rieffel and Van Daele [20], that $\mathscr{N}\left(W_{R}\right)$ and $\mathscr{M}$ coincide. With the same arguments we obtain $\mathscr{N}\left(W_{L}\right)=\mathscr{M}^{\prime}$. So we have duality for wedges and, since $\mathcal{N}\left(D_{a, b}\right)$ coincides with 
the Bisognano-Wichmann-net, one has duality also for this net [1] provided $\Omega$ is cyclic for $\mathscr{N}\left(D_{a, b}\right)$.

Remark. In this section we assumed that the algebra $\mathscr{M}$ is invariant under the action of the semi-group $\{\operatorname{ad} U(a), a \in W\}$. If, instead, one takes an algebra $\mathscr{M}$ invariant under the semi-group $\left\{\operatorname{ad} U(a), a \in V^{+}\right\}$then the modular group acts as dilatation, because the scaling for both lightcone coordinates is the same. This dilatation exists only as an automorphism on the translations and not as local transformations on the algebras $\mathscr{M}(O)$. If however, the commutant of $\mathscr{M}\left(V^{+}\right)$ coincides with $\mathscr{M}\left(V^{-}\right)$then the modular transformations also act local on the algebras. This latter case appears in the theory of free massless fields.

\section{The CPT-Theorem in the Theory of Local Observables in Two Dimensions}

Up to now we started with only one von Neumann-algebra, which had several properties listed in Sect. III. Out came a representation of the Poincaré-group and a CPT-operator; and eventually a local structure with the amazing result that the representation of the Poincare-group is an extension of the given representation of the translation group.

Next we start with a net of local observables $\left\{\mathscr{M}(O), \mathscr{M}, U(a) \mathbf{R}^{2}, \Omega, \mathscr{M}\right\}$, i.e. to every double cone $D_{a, b} \subset \mathbf{R}^{2}$ we have a von Neumann-algebra $\mathscr{M}\left(D_{a, b}\right)$ on $\mathscr{H}$ fulfilling isotony and covariance under translations. In addition, we assume $U(c) \mathscr{M}\left(D_{a, b}\right) U^{*}(c)=\mathscr{M}\left(D_{a+c, b+c}\right)$, where $U(a)$ is a continuous representation of the translation group fulfilling spectrum condition and which has $\Omega$ as an invariant vector. Furthermore, we assume that this net fulfills the locality condition, that means, if $D_{a, b}$ and $D_{c, d}$ are spacelike to each other, then $\mathscr{M}\left(D_{a, b}\right)$ and $\mathscr{M}\left(D_{c, d}\right)$ are commuting von Neumann-algebras. $\mathscr{M}$ is the von Neumann-algebra generated by all $\mathscr{M}\left(D_{a, b}\right)$ and we assume that $\Omega$ is a cyclic vector for $\mathscr{M}$.

Let now $\mathscr{M}\left(W_{R}\right)$ or $\mathscr{M}\left(W_{L}\right)$ respectively, be the smallest von Neumann-algebra containing all $\mathscr{M}\left(D_{a, b}\right)$ with $D_{a, b} \subset W_{R}$ or $D_{a, b} \subset W_{L}$ respectively.

Using Lemma III.4 we see that $\Omega$ is a cyclic and separating vector for $\mathscr{M}\left(W_{R}\right)$ and also for $\mathscr{M}\left(W_{L}\right)$. Starting with $\mathscr{M}\left(W_{R}\right)$ we are in a situation described in Sect. III, i.e. with these methods we obtain a modular operator $\Delta_{R}$, a modular conjugation $J_{R}$, and, using the construction described in Sect. III, a Bisognano-Wichmann-net $\mathscr{N}_{R}\left(D_{a, b}\right)$ fulfilling the duality condition. Since $\mathscr{M}\left(W_{L}\right)$ commutes with $\mathscr{M}\left(W_{R}\right)$ one has $\mathscr{M}\left(D_{a, b}\right) \subset \mathscr{N}_{R}\left(D_{a, b}\right)$ so that the originally given net $\mathscr{M}\left(D_{a, b}\right)$ is contained in the Bisognano-Wichmann-net $\mathscr{N}_{R}\left(D_{a, b}\right)$,

$$
\mathscr{M}\left(D_{a, b}\right) \subset \mathscr{N}_{R}\left(D_{a, b}\right) .
$$

For this construction we had a choice. Instead of starting with the vonNeumannalgebra $\mathscr{M}\left(W_{R}\right)$ we could have started with $\mathscr{M}\left(W_{L}\right)$. In this case we obtain a second set $\left\{\Delta_{L}, J_{L}, \mathscr{N}_{L}\left(D_{a, b}\right)\right\}$. Here $\mathscr{N}_{L}\left(D_{a, b}\right)$ is again a Bisognano-Wichmann-net fulfilling duality. Each of the two nets contain the original net $\mathscr{M}\left(D_{a, b}\right)$.

Looking at examples in the standard Minkowski-space one might expect that there are some examples giving rise to two different Bisognano-Wichmann-nets. Take, for instance, a Poincaré-covariant Wightman field theory with a finite number 
of components, but which is no longer covariant when dropping some of them. If one starts with the covariant field then, by the construction of Bisognano and Wichmann, who used Jost's CPT-theorem [14], one obtains a local net $\mathscr{M}(O)$ such that $\mathscr{M}\left(W_{R}\right)^{\prime}=\mathscr{M}\left(W_{L}\right)$. But if we drop some of the components one loses Lorentzcovariance and in this case the identity of $\mathscr{M}(W(R))^{\prime}$ and $\mathscr{M}\left(W_{L}\right)$ is doubtful.

We still have to justify the statement that the operator $J$ can be identified with the CPT-operator. For these investigations we shall restrict ourselves to the case where one starts from the wedge $W_{R}$. We know that $J_{R}$ acts as a total reflection on the algebras $\mathcal{N}_{R}\left(D_{a, b}\right)$. In order to show that $J_{R}$ also acts as charge conjugation we assume that the local rings are structured in such a way that we can write $\mathscr{M}\left(D_{a, b}\right)=\sum_{j} \mathscr{M}_{j}\left(D_{a, b}\right)$ and that we have a compact gauge group $G$ acting on $\mathscr{M}$ by automorphism $\beta_{\gamma}, \gamma \in G$ which map every $\mathscr{M}\left(D_{a, b}\right)$ onto itself. In particular we assume that the following equation holds:

$$
\beta_{\gamma} \mathscr{M}_{i}\left(D_{a, b}\right)=\sum c_{i, j}(\gamma) \mathscr{M}_{j}\left(D_{a, b}\right),
$$

where $c_{i, j}(\gamma)$ is a finite dimensional representation of $G$. This formula means, in detail, that to every $A_{i} \in \mathscr{M}_{i}\left(D_{a, b}\right)$ we can find $A_{j} \in \mathscr{M}_{j}\left(D_{a, b}\right)$, not depending on $\gamma$, with

$$
\beta_{\gamma}\left(A_{i}\right)=\sum_{j} c_{i . j}(\gamma) A_{j}
$$

In this formula the local observables are those operators which are invariant under the gauge transformations. Also the vacuum state is invariant under these transformations. Since $\beta_{\gamma}$ is a local gauge it extends to $\mathscr{M}\left(W_{R}\right)$ and to its commutant. From this we see that $\beta_{\gamma}$ commutes with the modular automorphisms and the modular conjugation. Consequently also the local net $\mathscr{N}_{R}\left(D_{a, b}\right)$ has the same structure as $\mathscr{M}\left(D_{a, b}\right)$. From these remarks we obtain for the conjugated quantities $j_{R}\left(\mathscr{N}_{R, i}\left(D_{a, b}\right)\right)=J_{R} \mathscr{N}_{R, i}\left(D_{a, b}\right) J_{R}$ the equation

$$
\beta_{\gamma} J_{R}\left(\mathscr{N}_{R, i}\left(D_{a, b}\right)\right)=\sum_{j} \bar{c}_{i, j}(\gamma) j_{R}\left(\mathscr{N}_{R, j}\left(D_{a, b}\right)\right)
$$

We learn from this equation that $J_{R}$ acts on the local net $\mathcal{N}_{R, j}\left(D_{a, b}\right)$ also as charge-conjugation. The best example is a field carrying a standard charge. Here one has $\beta_{\gamma}\left(\mathscr{N}_{R, i}\left(D_{a, b}\right)\right)=e^{i \varphi(\gamma, i)} \mathcal{N}_{R, i}\left(D_{a, b}\right)$ while the conjugation $J_{R}$ changes the face factor into it's inverse. This means that the sign of the charge is reversed.

The case of the Fermi-fields can be treated as it has been done by Bisognano and Wichmann [2] and we don't have to repeat it here. From this discussion we see that $J_{R}$ also acts as a charge-conjugation so that, altogether, it can be viewed as the CPT-operator.

In the case of $\mathscr{M}\left(W_{R}\right)^{\prime} \neq \mathscr{M}\left(W_{L}\right)$ the operators $J_{R}$ and $J_{L}$ are inducing the same automorphism on the translation group, which implies that $J_{R} J_{L}^{-1}=J_{R} J_{L}$ commutes with the translations, i.e. we have

$$
\left[J_{R} J_{L}, U(a)\right]=\left[J_{L} J_{R}, U(a)\right]=0 .
$$

In the same manner $\Delta_{R}^{t t}$ and $\Delta_{L}^{-t}$ induce the same automorphism on the translations, which implies

$$
\left[\Delta_{R}^{u t} \Delta_{L}^{t t}, U(a)\right]=\left[\Delta_{L}^{u t} \Delta_{R}^{u t}, U(a)\right]=0
$$


If we have the situation $\mathscr{M}\left(W_{L}\right)^{\prime} \neq \mathscr{M}\left(W_{R}\right)$ then $\mathscr{M}\left(W_{R}\right)^{\prime}$ and $\mathscr{M}\left(W_{L}\right)^{\prime}$ do not commute with each other. This implies that the two nets $\left\{\mathscr{N}_{R}\left(D_{a, b}\right)\right\}$ and $\left\{\mathscr{N}_{L}\left(D_{a, b}\right)\right\}$ are not relatively local to each other. Since we started from a local net $\left\{\mathscr{M}\left(D_{a, b}\right)\right\}$, the two algebras $\mathscr{M}\left(W_{R}\right)$ and $\mathscr{M}\left(W_{L}\right)$ commute which implies that

$$
\mathscr{N}_{R}\left(D_{a, b}\right) \subset \mathscr{N}_{L}\left(D_{c, d}\right)^{\prime} \text { for } D_{a, b} \text { to the right of } D_{c, d} \text {. }
$$

But it is not true for the reverse order, i.e. if $D_{a, b}$ is to the left of $D_{c, d}$. If, again, $\mathscr{M}\left(W_{R}\right)^{\prime} \neq \mathscr{M}\left(W_{L}\right)$ then $\left\{\mathscr{N}_{R}\left(D_{a, b}\right) U(a), U_{R}(\Lambda)\right\}$ and $\left\{\mathscr{N}_{L}\left(D_{a, b}\right), U(a), U_{L}(\Lambda)\right\}$ are not the only covariant local nets fulfilling duality, which one can construct from the local net $\left\{\mathscr{M}\left(D_{a, b}\right), U(a)\right\}$. We show

IV.1. Theorem. Assume that we start from a local net $\left\{\mathscr{M}\left(D_{a, b}\right), U(a)\right\}$ fulfilling the assumptions described above. Assume in addition that $\mathscr{M}\left(W_{L}\right)^{\prime} \neq \mathscr{M}\left(W_{R}\right)$, then there exist two sequences of different covariant local nets fulfilling duality $\left\{\mathscr{N}_{R}^{i}\left(D_{a, b}\right)\right.$, $\left.U(a) U_{R}^{i}(\Lambda)\right\}$ and $\left\{\mathscr{N}_{L}^{i}\left(D_{a, b}\right), U(a) U_{L}^{i}(\Lambda)\right\} i \in \mathbf{Z}$, such that

1)

$$
\begin{gathered}
\mathscr{N}_{R}^{0}\left(D_{a, b}\right)=\mathscr{N}_{R}\left(D_{a, b}\right), \\
\mathscr{N}_{L}^{0}\left(D_{a, b}\right)=\mathscr{N}_{L}\left(D_{a, b}\right),
\end{gathered}
$$

where $\mathscr{N}_{R}\left(D_{a, b}\right)$ and $\mathcal{N}_{L}\left(D_{a, b}\right)$ are the two nets constructed before.

2)

3)

$$
\begin{aligned}
\mathscr{N}_{R}^{i}\left(W_{R}\right) & \supset \mathscr{N}_{R}^{i+1}\left(W_{R}\right), \\
\mathcal{N}_{L}^{i}\left(W_{R}\right) & \supset \mathscr{N}_{L}^{i+1}\left(W_{R}\right), \quad i \in \mathbf{Z}
\end{aligned}
$$

$$
\mathscr{N}_{L}^{i}\left(W_{R}\right) \supset \mathscr{N}_{R}^{i}\left(W_{R}\right), \quad i \in \mathbf{Z} .
$$

Proof. The construction we will use has been introduced by V.F.R. Jones [13] and by R. Longo [15] for the study of subfactors. Let $W$ be the operator $J_{R} J_{L}$, which is unitary. Moreover, one has $W^{*}=W^{-1}=J_{L} J_{R}$. Define

$$
\begin{aligned}
& \mathscr{N}_{R, L}^{0}\left(W_{R}\right)=\mathscr{N}_{R, L}\left(W_{R}\right) \quad \text { and } \\
& \mathscr{N}_{R, L}^{i}\left(W_{R}\right)=W^{i} \mathscr{N}_{R, L}\left(W_{R}\right) W^{-i}
\end{aligned}
$$

Since $\mathscr{N}_{R}$ and $\mathscr{N}_{L}$ are constructed from a local net $\left\{\mathscr{M}\left(D_{a, b}\right)\right\}$ one has

$$
\mathscr{N}_{L}\left(W_{R}\right)=\mathscr{N}_{L}\left(W_{L}\right)^{\prime} \supset \mathcal{N}_{R}\left(W_{R}\right)
$$

which implies 3) by the defining Eq. (23). Statement 1 ) is only a matter of definition.

Next we show 2). By definition of the modular conjugations we have $J_{L} \mathscr{N}_{L}\left(W_{L}\right)^{\prime} J_{L}=\mathscr{N}_{L}\left(W_{L}\right)$ and $J_{R} \mathscr{N}_{R}\left(W_{R}\right)^{\prime} J_{R}=\mathscr{N}_{R}\left(W_{R}\right)$. Since we also have $\mathscr{N}_{R}\left(W_{R}\right) \subset \mathcal{N}_{L}\left(W_{L}\right)^{\prime}$ and $\mathcal{N}_{L}\left(W_{L}\right) \subset \mathcal{N}_{R}\left(W_{R}\right)^{\prime}$ we obtain $W \mathscr{N}_{R}\left(W_{R}\right) W^{-1} \subset \mathscr{N}_{R}\left(W_{R}\right)$. By Eq. (23) we obtain the line of 2). But the same argument gives $W \mathscr{N}_{L}\left(W_{L}\right) W \subset \mathscr{N}_{L}\left(W_{L}\right)$. Passing to the commutants and using again Eq. (23) we obtain the second line of 2). It remains to show that the $\mathscr{N}_{R, L}^{i}\left(D_{a, b}\right)$ form a local net. From $\mathscr{N}_{R, L}^{i}\left(W_{R}\right)=W^{i} \mathcal{N}_{R, L}\left(W_{R}\right) W^{-i}$ we obtain, by passing to the commutant, $\mathscr{N}_{R, L}^{i}\left(W_{L}\right)=W^{i} \mathcal{N}_{R, L}\left(W_{L}\right) W^{-i}$, and hence $\mathscr{N}_{R, L}^{i}\left(D_{a, b}\right)=W^{i} \mathcal{N}_{R, L}\left(D_{a, b}\right) W^{-i}$. Since one has $W \Omega=\Omega$ it follows $W^{i} \Omega=\Omega$. This implies that $\Omega$ is a cyclic and separating vector for $\mathscr{N}_{R, L}^{i}\left(D_{a, b}\right)$, since it is cyclic and separating for $\mathscr{N}_{R, L}\left(D_{a, b}\right)$ and $W^{i}$ is unitary. Finally, since $W$ commutes with $U(a)$, all these local nets are transformed 
by the same representation of the translations. The representation of the Lorentzgroup is given by

$$
U_{R, L}^{\prime}(\Lambda)=W^{i} U_{R, L}(\Lambda) W^{-i}
$$

Finally it remains to show that all these local nets are different. Assume first we have $\mathscr{N}_{R}^{i}\left(D_{a, b}\right)=\mathscr{N}_{R}^{j}\left(D_{a, b}\right), i \neq j$ for all double cones then we conclude $N_{R}^{i}\left(W_{R}\right)=\mathscr{N}_{R}^{j}\left(W_{R}\right)$. Using the first line of 2) we obtain $\mathscr{N}_{R}^{0}\left(W_{R}\right)=\mathscr{N}_{R}^{1}\left(W_{R}\right)$. This equation implies $J_{L} \mathscr{N}_{R}^{0}\left(W_{R}\right) J_{L}=J_{R} \mathscr{N}_{R}^{1}\left(W_{R}\right) J_{R}=J_{R} \mathscr{N}_{R}^{0}\left(W_{R}\right) J_{R}$. From this we obtain

$$
\mathscr{N}_{L}^{0}\left(W_{L}\right)=J_{L} \mathscr{N}_{L}^{0}\left(W_{L}\right)^{\prime} J_{L} \supset J_{L} \mathscr{N}_{R}^{0}\left(W_{R}\right) J_{L}=J_{R} \mathscr{N}_{R}^{0}\left(W_{R}\right) J_{R}=\mathscr{N}_{R}^{0}\left(W_{R}\right)^{\prime}
$$

This contradicts the assumption. If we assume $\mathscr{N}_{L}^{i}\left(D_{a, b}\right)=\mathscr{N}_{L}^{i}\left(D_{a, b}\right) i \neq j$ then a similar argument leads to the same conclusion.

Next assume $\mathscr{N}_{R}^{i}\left(D_{a, b}\right)=\mathscr{N}_{L}^{i}\left(D_{a, b}\right)$ for all double cones. If we assume $i \leqq j$ then we have $\mathscr{N}_{L}^{j}\left(W_{L}\right)^{\prime}=\mathscr{N}_{R}^{i}\left(W_{R}\right) \subset \cdots \subset \mathscr{N}_{R}^{j}\left(W_{R}\right)$. From this we obtain that the net $\mathscr{N}_{R}^{i}\left(D_{a, b}\right)$ fulfills duality and consequently also the net $\mathscr{N}_{R}^{0}\left(D_{a, b}\right)$. This contradicts the assumption. If we have $i>j$ then we only have to interchange the right and the left wedge in order to obtain the same conclusion.

The original net $\mathscr{M}\left(D_{a, b}\right)$ is contained in $\mathscr{N}_{R, L}\left(D_{a, b}\right)$ but in general it will not be contained in $\mathscr{N}_{R, L}^{i}\left(D_{a, b}\right)$ for $i \neq 0$.

Finally, we consider the case of starting from a Lorentz-covariant net $\left\{\mathscr{M}\left(D_{a, b}\right)\right.$, $U(a), V(\Lambda)\}$ fulfilling the standard assumptions. Then we obtain:

IV.2. Theorem. Let $\left\{\mathscr{M}\left(D_{a, b}\right), U(a), V(\Lambda)\right\}$ be a (two-dimensional) Lorentz-covariant local net and let $\left\{\mathcal{N}_{R, L}^{i}\left(D_{a, b}\right) U(a) U_{R, L}^{i}(\Lambda)\right\}$ be the derived covariant nets (see Theorem IV.2) then one has

(i) $V(\Lambda)$ commutes with all $U_{R, L}^{i}\left(\Lambda^{\prime}\right), i \in \mathbf{Z}$ and with all modular conjugations $J_{R, L}^{i} i \in \mathbf{Z}$

(ii) $\left\{\mathscr{N}_{R, L}^{i}\left(D_{a, b}\right), U(a), V(\Lambda)\right\}$ is again a covariant local net, i.e. one has

$$
V(\Lambda) \mathcal{N}_{R, L}^{i}\left(D_{a, b}\right) V^{-1}(\Lambda)=\mathcal{N}^{i}\left(D_{\Lambda a, \Delta b}\right)
$$

(iii) The group $X_{R L}^{i}(\Lambda)=U_{R, L}^{i}(\Lambda) V^{*}(\Lambda)$ defines local gauges for the algebras $\mathscr{N}_{R, L}^{i}\left(D_{a, b}\right)$, i.e. one has

$$
\operatorname{ad} X_{R L}^{i}(\Lambda) \mathscr{N}_{R, L}^{i}\left(D_{a, b}\right)=\mathscr{N}_{R, L}^{i}\left(D_{a, b}\right) \quad i \in \mathbf{Z}
$$

Proof. (i) Since we have $\mathscr{M}\left(W_{R}\right)=\mathscr{N}_{R}\left(W_{R}\right)$ it follows that $C(\Lambda)$ is an invariance-group of $\mathcal{N}_{R}\left(W_{R}\right)$. This implies by [6] Theorem 3.2.18, that $V(\Lambda)$ commutes with $\Delta_{R}$ and $J_{R}$. Since $U_{R}(\Lambda)$ is derived from $\Delta_{R}$ it follows that $V(\Lambda)$ commutes with all $U_{R}\left(\Lambda^{\prime}\right)$. Replacing $W_{R}$ by $W_{L}$ we obtain by the same argument that $V(\Lambda)$ commutes with $J_{L}$ and $U_{L}\left(\Lambda^{\prime}\right)$. But this implies that $V(\Lambda)$ commutes with $W=J_{R} J_{L}$. Since all other modular operators and modular conjugations are obtained by transforming $U_{R}$ and $U_{L}$ or $J_{R}$ and $J_{L}$ respectively, by powers of $W$ we see that all of these operators commute with $V(\Lambda)$.

(ii) From ad $V(\Lambda) \mathscr{N}_{R}\left(W_{R}\right)=\mathscr{N}_{R}\left(W_{R}\right)$ one has ad $V(\Lambda) \mathscr{N}_{R}\left(W_{R}\right)^{\prime}=\mathscr{N}_{R}\left(W_{R}\right)^{\prime}$. Since $V(\Lambda)$ induces the correct automorphism on the translation group it follows 
from the definition of $\mathscr{N}_{R}\left(D_{a, b}\right)$ that also $V(\Lambda)$ transforms these algebras correctly, i.e.

$$
\operatorname{ad} V(\Lambda) \mathscr{N}_{R}\left(D_{a, b}\right)=\mathscr{N}_{R}\left(D_{\Lambda a, \Lambda b}\right)
$$

Replacing $W_{R}$ by $W_{L}$ we obtain the corresponding statement for $\mathscr{N}_{L}\left(D_{a, b}\right)$. Finally, since $\mathscr{N}_{R, L}^{i}\left(D_{a, b}\right)$ is obtained by transforming $\mathscr{N}_{R, L}\left(D_{a, b}\right)$ with $W^{i}$ we obtain again that $V(\Lambda)$ transforms these algebras in the correct manner.

(iii) From the equation

$$
U_{R, L}^{\prime}(\Lambda) \mathcal{N}_{R, L}^{i}\left(D_{a, b}\right) U_{R L}^{i}(\Lambda)^{*}=V(\Lambda) \mathscr{N}_{R L}^{i}\left(D_{a, b}\right) V^{*}(\Lambda)
$$

we obtain

$$
X_{R, L}^{i}(\Lambda) \mathcal{N}_{R, L}^{i}\left(D_{a, b}\right) X_{R L}^{i}(\Lambda)^{*}=\mathcal{N}_{R, L}^{i}\left(D_{a, b}\right)
$$

which means that $X_{R, L}^{i}(\Lambda)$ defines a group of local gauges for the algebras $\mathcal{N}_{R, L}^{i}\left(D_{a, b}\right)$.

\section{References}

1. Bisognano, J., Wichmann, E. H.: On the duality for a hermitian scalar field. J. Math. Phys. 16, 985-1007 (1975)

2. Bisognano, J., Wichmann, E. H.: On the duality for quantum fields. J. Math. Phys. 17, 303-321 (1975)

3. Borchers, H.-J.: Local Rings and the Connection of Spin with Statistics. Commun. Math. Phys. 1, 281-307 (1965)

4. Borchers, H.-J.: On the converse of the Reeh-Schlieder Theorem. Commun. Math. Phys. 10, 269-273 1968

5. Borchers, H.-J.: Translation Group and Modular Automorphisms for Local Regions. Commun. Math. Phys. 132, 189-199 (1990)

6. Bratteli, O., Robinson, D. W.: Operator Algebras and Quantum Statistical Mechanics I. New York, Heidelberg, Berlin: Springer 1979

7. Brehmermann, H. J., Oehme, R., Taylor, J. G.: Proof of dispersion relation in quantized field theories. Phys. Rev. 109, 2178-2190 (1985)

8. Browder, F.: On the "Edge of the Wedge" Theorem. Canad, J. Math. 15, 125-131 (1963)

9. Buchholz, D., Fredenhagen, K.: Locality and the structure of particle states. Commun. Math. Phys. 84, 1-54 (1982)

10. Doplicher, S., Haag, R., Roberts, J. E.: Local Observables and Particle Statistics I. Commun. Math. Phys. 23, 199-230 (1971)

11. Doplicher, S., Haag, R., Roberts, J. E.: Local Observables and Particle Statistics II. Commun. Math. Phys. 35, 49-85 (1974)

12. Fredenhagen, K.: On the existence of antiparticles. Commun. Math. Phys. 79, 141-151 (1981)

13. Jones, V. F. R.: Index for subfactors. Invent. Math. 72, 1-25 (1983)

14. Jost, R.: Eine Bemerkung zum CTP Theorem. Helv. Phys. Acta 30, 409-416 (1957)

15. Longo, R.: Index for Subfactors and Statistics of Quantum Fields I. Commun. Math. Phys. 126, 217-247 (1989)

16. Lüders, G.: On the equivalence of invariance under time reversal and under particleantiparticle conjugation for relativistic field theories. Danske Vidensk. Selskab, Mat.-fysiske Meddelelser 28, (5), 1-17 (1951)

17. Lüders, G.: Proof of the CTP Theorem. Ann. Phys. 2, 1-15 (1957)

18. Monoharan: A. C.: Some Considerations on Configuration Space Analyticity in General Quantum Field Theory. Lectures in Theoretical Physics, Boulder (1988), Vol XI-D Mathematical Methods in Theoretical Physics, Mahanthappa, K., Brittin, W. E. (ed.), New York, London, Paris: Gordon and Breach 1969 
19. Pauli, W.: Exclusion Principle, Lorentz Group and Reflection of Space-Time and Charge. Niels Bohr and the Development of Physics, Pauli, W., Rosenfeld, I., Weisskopf, V. (eds.). London: Pergamon Press 1955

20. Rieffel, M., Van Daele, A.: The commutation theorem for tensor products of von Neumann algebras. Bull. Lond. Math. Soc. 7, 257-260 (1975)

21. Rigotti, C.: Remarks on the Modular Operator and Local Observables. Commun. Math. Phys. 61, 267-273 (1978)

22. Schwinger, J.: The Theory of Quantized Fields I. Phys. Rev. 82, 914-927 (1951)

23. Streater, R., Wightman, A. S.: PCT, Spin and Statistics, and all that. New York, Amsterdam: Benjamin, W. A. 1964

24. Takesaki, M.: Tomita's theory of modular Hilbert algebras and its applications. Lecture Notes in Mathematics, vol. 128. Berlin, Heidelberg, New York: Springer 1970

25. Tomita, M.: Standard forms of von Neumann algebras. Fifth functional analysis symposium of Math. Soc. of Japan, Sendai (1967)

26. Wightman, A. S.: Quantum field theory in terms of vacuum expectation values. Phys. Rev. 101,860 (1956)

Communicated by H. Araki 